Carex Pseudocyperus L. Lago Pudro bei Pergine S. (Roveredo Cristoforis, Lago di Garda Precht).

- fliformis L. Lago Serraja: Piné G. (Bondone Ambrosi, Tonale Rota, V. Ledro Facchini).

- litigosa Chaub. V. Vestino P. N. f. d. T.

Setaria ambigua Guss. Trento G. N. f. d. T.

Phleum asperum Villars V. di Non G. V. Vestino P. (Trento Heuf-

ler, Roveredo Cristoforis, Canal S. Bovo, Arco fl. Ambrosi). Agrostis rupestris All. Campiglio S. V. Genova G. S. (Valsugana

Ambrosi, Fassa, Fiemme Facchini, Baldo Pollini, Val di Non Leybold).

- gigantea Roth V. di Ledro P. N. f. d. T.

Stipa capillata L. Trento G. (Fiemme Facchini).

Avena amethystina Clairv. Bondone S. (Baldo, Fassa, Giudicarie Facchini).

- subspicata Clairv. Tonale G. S. (Valsugana Paterno, Fassa Facchini).

- Hostii Boiss. V. Ledro P. N. f. d. T.

Danthonia provincialis DC. Trento: Calisio G., V. Vestino P. (Roveredo: Cengialto, Trembilleno; Lago di Garda Facchini).

Poa sudetica Haenke Bondone G. (Fassa, Fiemme, Giudicarie, Valsugana Facchini).

Festuca ciliata Denth. Calliano G. (Riva, Arco Facchini).

Bromus erectus Huds. (stenophyllus Link.) Trento S. N. f. d. T.

Triticum glaucum Desf. Trento S. V. Vestino P. (Roveredo Cristoforis).

Schliesslich erlaube ich mir, meinen Freunden Peter Porta und Heinrich Gelmi hiemit zu danken für ihre Bereitwilligkeit, mit der sie mir ihre für die Flora des Trentino so werthvollen Resultate mittheilten.

Trient, 20. Jänner 1881.

\title{
Ueber das Vorkommen ron
}

Crocus vermos Wulf, in den Sudeten.

Von E. Fiek.

Bei Besprechung des Crocus banaticus Heuffel in den "Vegetations-Verhältnissen des mittleren Ungarn etc." (XXVII, 9) bemerkt Professor A. Kerner in Bezug auf dessen Verbreitung, dass er den karpathischen Gebirgen und deren Vorländern angehöre und besonders in den Ostkarpathen verbreitet sei. Ebenso erwähnt er des Vorkommens bei Neusobl, dem sich weitere Standorte in den westlichen Karpathen 
anreihen, wie er auch noch auf der Babiagóra in Westgalizien von Stein in Menge gefunden worden ist. Schlosser gibt Crocus auf Bergwiesen bei Sponau in Mähren an, wo die Pflanze auch später von Anderen gefunden wurde; leider habe ich nicht eruiren können, ob dieser Standort im Gebiete der Beskiden (Karpathen) oder in dem der Sudeten gelegen ist, da mir eine genaue Specialkarte von Mähren mangelt. Aus dem östlichsten Theile der Sudeten nennt Kerner (a. a. 0. S. 296) irrig noch einen Fundort für C. albiflorus Kit.: "Leobschütz-Troppau", nämlich den zwischen diesen beiden Städten gelegenen bei Braunsdorf.

Wimmer gibt in seiner Flora von Schlesien (ed. III, 1857) für Crocus vernus All. dort zwei Standorte an: "Braunsdorf bei Troppau nach v. Mückusch; Gr. Herlitz auf der Horzina-Wiese nach Mayer", eine Angabe, die in Garcke's verbreitete Flora übergegangen und noch in deren neuester Auflage enthalten ist. Wie mir schon früher mein mit der Flora dieses Theiles von Schlesien sehr vertrauter Freund Wetschky in Gnadenfeld mitgetheilt hatte, existirt in jener Gegend jedoch nur eine Stelle, an welcher diese Pflanze wächst, und die liegt eben zwischen den genannten beiden Dörfern Gr. Herlitz und Braunsdorf. Im Jahre 1879 lernte ich diesen, ungefähr 14 Kilometer von Troppau gelegenen Punkt kennen. Cracus vernus Wulfen (nicht $C$. albiflorus Kit.) bedeckt da zu Tausenden die buschigen Wiesen am Horzinabache, eine kleine Stunde vor dessen Einmündung in die Oppa, und gewährt mit seinen violetten Blüthen nebst der gleichfalls dort zahlreich vorkommenden Corydatis solida (L.) Sm. einen prächtigen, für die frühe Jahreszeit recht ungewohnten Anblick. Wahrscheinlich ist Mayer, der seiner Zeit Güterinspector in Gr. Herlitz war und von dem auch eine Anzahl Exemplare aus den ersten Jahren der um 1820 oder 21 erfolgten Entdeckung im Herbarium der Schles. Gesellschaft in Breslau vorbanden sind, der Entdecker dieser reizenden Frühlingspflanze für Schlesien.

Diesem, Jahrzehnte lang als einziges Vorkommen des Crocus in "Nord- und Mitteldeutschland" erwähnten Standorte fügt Wimmer noch einige andere hinzu: "Grafenort bei Habelschwerdt nach Boguslaw Fürst Radziwil; Grasgärten in Schmiedeberg nach Kaiser; Schreiberhau in Grasgärten nach Standfuss." Von den beiden ersteren konnte ich ebenso wenig, wie von der Angabe: „auf Wiesen bei Altwasser gegen Seitendorf" im Kreise Waldenburg "(Laugner) ermitteln, ob sie sich auf spontanes Auftreten beziehen; dagegen steht es fest, dass Crocus vernus Wulf. auch in den westlichen Sudeten (im Isergebirge) zu den wildwachsenden Pflanzen gehört und hier jedenfalls die Nordgrenze seiner Verbreitung erreicht.

Im Jahre 1875 hörte ich nämlich von einem mir befreundeten Lehrer, dass er Crocus zahlreich auf Wiesen in Schreiberhau gesehen habe, einem ungemein ausgedebnten Dorfe im Riesengebirge oder genauer im Riesen- und Isergebirge, da dasselbe im Zackenthale liegt, der Zacken aber bekanntlich Riesen- und Isergebirge scheidet. Auf diese Mittheilung hin besuchte ich im Frühjahre des folgenden 
Jahres jene Gegend, um Genaueres zu erfahren. Es wurde mir nicht schwer, nach jener Angabe die betreffende Stelle zu finden, zumal ich gerade zur Blüthezeit der Pflanze eintraf und diese sehr gesellig wachsende Zierde des Frühlings mir schon von weitem entgegen leuchtete. Nach und nach habe ich bei wiederholtem Besuch nicht weniger als 8, zum Theil eine Viertelstunde aus einander liegender Punkte aufgefunden, an denen der Crocus meist in grosser Menge die Wiesen bedeckt. Alle diese Stellen liegen durchschnittlich etwa 680 M. hoch in drei ziemlich flachen, von Wiesen erfüllten Senkungen, die sich vom schwarzen Berge, einer westlich rom Hochstein (im Isergebirge) sich erhebenden Kuppe ins Zackenthal hinabziehen. Während die obersten Partien dieses Berges mit Wald bedeckt sind, werden die Wiesen selbst nur von einzelnen Gehölzresten, meist jedoch von steinigen, den armen Borgbewohnern wohl nur kärglichen Ertrag liefernden Aeckern eingefasst. Obgleich einzelne Häuser zerstreut zwischen diesen Aeckern und Wiesen liegen, lässt die Art und Weise des Vorkommens unserer Pflanze ihr Auftreten an verschiedenen auseinander liegenden Stellen, den Gedanken an eine Verwildorung nicht aufkommen, ganz abgesehen davon, dass man nur bei wenigen dieser Häuschen Gärtchen antrifft, in denen noch dazu Crocus nicht einmal cultivirt wird. Auch wussten die Bewohner nur zu sagen, dass diese liebliche Frühlingsblume schon von jeher da gewesen sei, der verstorbene Lehrer Henne in Sehreiberhau sie auch nach zuverlässiger Mittheilung schon in don ersten Jahrzehnten unseres Jabrhunderts in gleicher Menge gekannt habe.

War ich nun auch von dem Indigenat unseres Crocus überzeugt, so musste der leiseste Zweifel schwinden, als es mir im vergangenen Frühjahre nach langem Suchen gelang, ihn nach Angabe des Revierförster Maiwald auch auf der, Schreiberhau entgegengesetzten (nördlichen) Seite des schwarzen Berges, im Thale des kleinen Zacken an grasigen, lichten Waldstellen des "Schooshübel", weit entfernt von menschlichen Wohnungen, zu finden.

Unsere Pflanze hat eine schön violette Corolle, deren Zipfel länglich-verkehrteiförmig, 2-3mal so lang als breit und concav sind, die Narbe ragt über die Antherenspitzen hinaus, und zwar bei den Troppaner Exemplaren relativ etwas mehr als bei denen von Schreiberhau. Auch im Uebrigen stimmt sie mit den von verschiedenen Orten aus den Ostkarpathen erhaltenen genau überein und ist somit identisch mit Crocus banaticus Heuffel. Diese Form ist aber nach meiner Ueberzeugung ziemlich identisch mit $C$. vernus Wulfen, da die von Kerner (a, a. O. S. 296) hervorgehobenen Unterschiedo zwischon beiden durchaus nicht constant sind. Die Blätter pflegen bei unserer Sudetenpflanze allerdings vorherrschend verlängert lineal-lanzettlich oder eigentlich lanzettlich-lineal zu sein (C. banaticus). man findet neben solchen aber auch immer zahlreiche Individuen, die fast vollig lineale Blätter besitzen (C. vernus) und ebenso nicht wenige Uebergänge zwischen beiden Formen. Der Schlund der Corolle soll ferner bei C. banaticus kahl sein, gegenüber dem daselbst bärtigen $C$. vernus. 
Unsere Exemplare zeigen aber an der Einfügnngsstelle der Staubblätter bald zahlreiche, bald ganz vereinzelte hyaline Papillen, die zwar leicht zu übersehen sind, aber selten oder nie völlig fehlen. Ein Gleiches gilt endlich von den für $C$. vernus angegebenen „feinflaumigen" Staubfäden. Sowohl die Troppauer wie Pflanze von Schreiberhau ist deshalb mit Crocus vernus Wulfen zu bezeichnen.

Hirschberg in Schlesien, den 4. Februar 1881.

\section{Pugillus plantarum novarum vel minus recte cognitarum.}

\section{Auctore Michaeli Gandoger.}

\section{(Fortsetzung.)}

Grex Aegilops triaristatae Willd. sp. IV. 943.

Aegilops calida Gdgr. Culmis diffuso-procumbentibus, dein rigidiuscule ascendentibus; foliis subglabris, rectis; vaginis subhirtellis, ad oram longe copioseque ciliatis; spica obovata; glumis apice haud contractis, a $1 / 2$ sup. usque ad basin longe abundeque ciliatam attenuatis; aristis $46^{2} \mathrm{~mm}$. longis, superne violaceis.

Hab. Italia orient. in monte Brisighella prope Faenza (L. Caldesi).

Vix pedalis, e basi ramosa diffuso-erecta; folia glaucescentia, $2 \mathrm{~mm}$. lata.

Aegilops algeriensis Gdgx. - Exs. Fragm. Fl. alger. exs. 2. Série, Nr. 398! Culmis basi tortuosis, deinde rectiuscule erectis; foliis inferioribus pilosis, superioribus antem subglabris, patulis; vaginis sat villosis, ad oram abunde villosis; spica oblonga; glumis apice subcontractis, a $1 / 3$ part. inf. ad basin longe parceque villosam sat attenuatis; aristis $35-37 \mathrm{~mm}$. longis, omnino flavescentibus.

Hab. Algeria, in incultis circa Constantine (Choulette).

Praecedento subrigidior, folia magis pilosa et spica elongata.

Aegilops viridescens Gdgr. Culmis basi geniculatis, deinde erecto-flexuosis; foliis inferioribus vix pubescentibus, superioribus vero subglaberrimis, rectis; vaginis omnibus glabris, ad oram sat copiose ciliatis; spica ovoidea, glumis superne breviter attenuatis, a 1/2 part. super. ad basin usque nunquam ciliatam abrupte contractis; aristis $45-47 \mathrm{~mm}$. longis, apice subviolaceis. dièr e).

Hab. Gallia, Hérault pone Les Ares (Verriet-Litar-

Glumae antecedentibus minus hirsutae, inferior $10 \mathrm{~mm}$. longa. Aegilops croatica Gdgr. Culmis basi subineurvis, deinde rigidoerectis; foliis inferioribus subhirsutis, superioribus fere glabris, erectis; vaginis glaberrimis, ad oram vix ciliatis; spica lineari-oblonga; glumis apice in $1 / 3$ part. super. sensim attenuatis, a $1 / 3$ part. infer. 